\title{
Staphylococcus epidermidis biofilms induce lower complement activation in neonates as compared with adults
}

\author{
Hildegunn N. Granslo ${ }^{1,2}$, Claus Klingenberg ${ }^{1,2}$, Elizabeth A. Fredheim ${ }^{1,2}$, Ganesh Acharya ${ }^{3,4}$, Tom Eirik Mollnes ${ }^{5,6}$ and \\ Trond Flægstad ${ }^{1,2}$
}

BACKGROUND: Staphylococcus epidermidis (SE) is an important cause of late-onset sepsis in neonates. SE frequently produces a polysaccharide intercellular adhesin (PIA) biofilm, important in the pathogenesis of these infections. Little is known about how the neonatal innate immune system reacts to SE biofilm-associated infections. Our hypothesis was that SE biofilms induce a lower complement activation in neonates as compared with adults.

METHODS: Cord blood from term infants $(n=15)$ and blood from adults $(n=6)$ were studied in an ex vivo whole-blood sepsis model. A PIA biofilm-producing strain (SE1457) and its isogenic mutant (M10), producing a non-PIA biofilm, were used.

RESULTS: Both SE biofilms induced stronger complement activation in adult than in cord blood $(P \leq 0.033)$. We found lower levels of antibodies toward both PIA $(P=0.002)$ and the whole bacterium $(P=0.001)$ in cord vs. adult blood. By contrast, the interleukin-8 (IL-8) and IL-6 secretion were higher in cord than in adult blood $(P \leq 0.002)$. The PIA biofilm induced stronger complement activation than the non-PIA biofilm.

CONCLUSION: We conclude that the neonatal complement system exhibits a maturational deficiency. This may reduce the ability of neonates to combat biofilm-associated SE infections.

Staphylococcus epidermidis (SE) is the most prevalent patho-

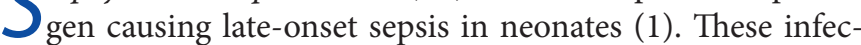
tions are seldom lethal, but they cause significant morbidity, especially in preterm infants $(1,2)$. SE infections are often associated with biofilm production on the surface of foreignbody implants $(3,4)$. Biofilms are adherent multicellular bacterial aggregates embedded in a self-produced extracellular matrix. The best-described matrix compound in SE biofilms is a $\beta$-(1,6)-linked $\mathrm{N}$-acetylglucosamine named polysaccharide intercellular adhesin (PIA) $(5,6)$.

SE and SE biofilms interfere with the host immune response at different levels. PIA biofilms inhibit the action of antimicrobial peptides (7), decrease neonatal inflammatory response (8), and decrease phagocytosis and degranulation by neutrophils (7). Biofilms may "decoy" antibodies and complement on the bacterial surface and thereby decrease opsonization and phagocytosis (9-11). The ability of biofilm-producing bacteria to avoid immune clearance and the general immaturity of the neonatal immune system (12) are postulated to increase the risk of neonatal SE sepsis (13). As compared with adults, neonates have a lower quantitative and qualitative complement activation $(14,15)$, reduced upregulation of cellular response (12), and an immature cytokine response pattern (16-19). Preterm infants demonstrate a markedly reduced capacity to upregulate oxidative burst in leukocytes in response to SE (20). However, some authors have reported that SE may induce a similar proinflammatory cytokine response in neonates and adults $(19,21)$, indicating a differential maturation of various parts of the neonatal innate immune system. Differences in maturation have also been described when challenging the innate immune system with meconium, for which the cytokine/chemokine response was at least as potent in neonates as in adults, whereas the complement activation was markedly lower in the neonates (22).

The immunological response to SE biofilms is very complex. We have previously shown that SE biofilms induce a strong complement response in blood from healthy adults (23), indicating a putative important role of the complement cascade in SE infections. To our knowledge, no previous studies have analyzed the complement response to SE biofilms in neonates. Our main hypothesis was that SE biofilms induce a lower complement activation in neonates as compared with adults. We therefore performed experiments in an ex vivo whole-blood model comparing the complement activation upon exposure to SE biofilms in cord blood vs. blood from adults. We also compared the complement activation in blood after exposure to PIA biofilm vs. a non-PIA biofilm.

\section{RESULTS}

Complement Activation in Cord Blood as Compared With Adult Blood Upon Stimulation With PIA Biofilm and Non-PIA Biofilm The PIA biofilm induced a higher activation of all complement activation products in adult $(n=6)$ blood as compared with cord $(n=15)$ blood; C1rs/C1 inhibitor complex, $P=0.018$;

'Department of Clinical Medicine, Pediatric Research Group, Faculty of Health Sciences, University of Troms $\varnothing$, Troms $\varnothing$, Norway; ${ }^{2}$ Department of Pediatrics, University Hospital of North Norway, Troms $\varnothing$, Norway; ${ }^{3}$ Department of Clinical Medicine, Women's Health and Perinatology Research Group, Faculty of Health Sciences, University of Troms $\varnothing$, Troms $\varnothing$, Norway; ${ }^{4}$ Department of Obstetrics \& Gynecology, University Hospital of Northern Norway, Tromsø, Norway; ${ }^{5}$ Department of Immunology and Transfusion Medicine, Nordland Hospital, Bodø, Norway; ${ }^{6}$ Department of Medical Biology, University of Tromsø, Tromsø, Norway. Correspondence: Hildegunn N. Granslo (hildegunn.granslo@uit.no) 


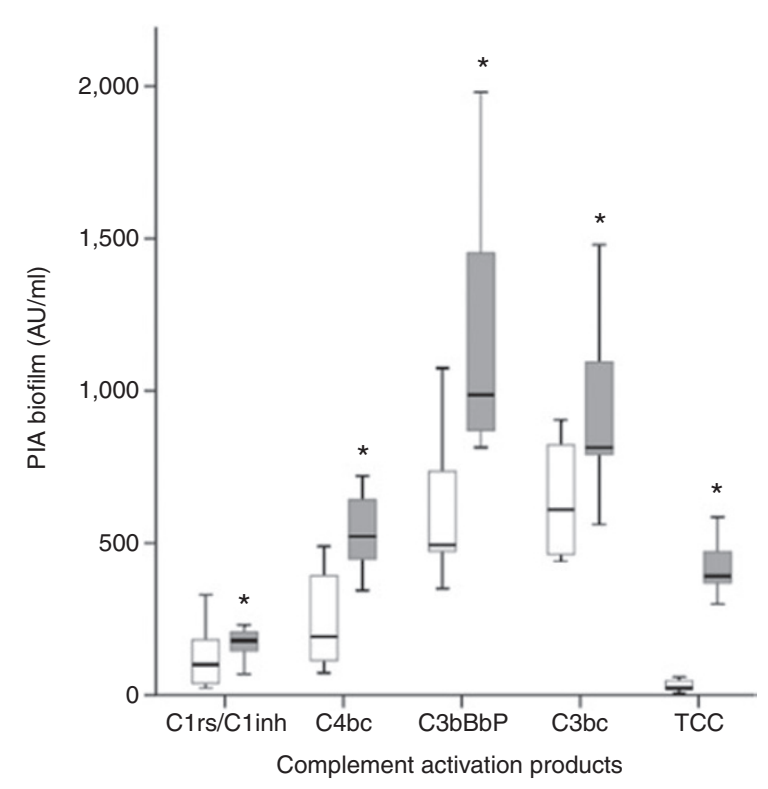

Figure 1. Comparison of the complement activation products in neonatal and adult blood after incubation with a PIA biofilm. The levels of the complement activation products $\mathrm{C} 1 \mathrm{rs} / \mathrm{C} 1$ inhibitor complex, $\mathrm{C} 4 \mathrm{bc}$, $\mathrm{C} 3 \mathrm{bBbP}, \mathrm{C} 3 \mathrm{bc}$, and terminal complement complex (TCC) were measured. White bars, neonate; gray bars, adult. *Statistically significant differences, $P<0.05$. PIA, polysaccharide intercellular adhesin.

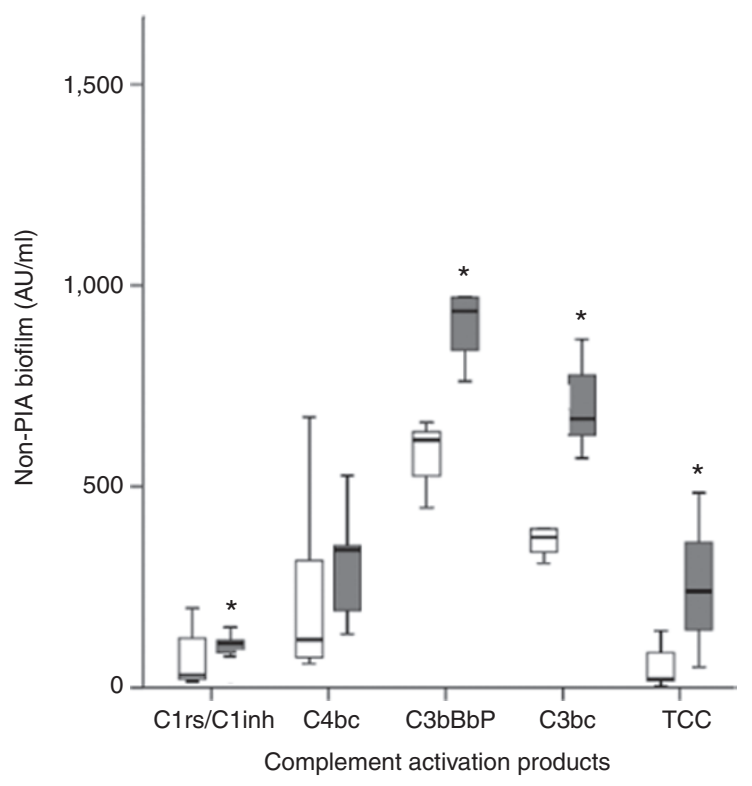

Figure 2. Comparison of the complement activation products in neonatal and adult blood after incubation with a non-PIA biofilm. The levels of the complement activation products $\mathrm{C} 1 \mathrm{rs} / \mathrm{C} 1$ inhibitor complex, $\mathrm{C} 4 \mathrm{bc}$, $\mathrm{C} 3 \mathrm{bBbP}, \mathrm{C} 3 \mathrm{bc}$, and terminal complement complex (TCC) were measured. White bars, neonate; gray bars, adult. * Statistically significant differences, $P<0.05$. PIA, polysaccharide intercellular adhesin.

C4bc, $P=0.008$; C3bBbP, $P=0.003$; C3bc, $P=0.012$; terminal complement complex, $P<0.001$ (Figure 1).

The non-PIA biofilm induced a higher activation of all complement activation products in adult blood $(n=6)$ as compared with cord blood $(n=15)$; C1rs/C1 inhibitor complex, $P=0.033$; C3bBbP, $P=0.003$; C3bc, $P=0.039$; terminal complement complex, $P=0.001$ (Figure 2). As compared with data for the PIA biofilm (Figure 1), the overall complement activation was lower when challenging with the non-PIA biofilm (Figure 2).

\section{Cytokine Secretion in Response to PIA and Non-PIA Biofilms}

Both the PIA and the non-PIA biofilms induced a significant secretion of interleukin-6 (IL-6) and IL-8 in cord blood as compared with the nonstimulated control (Figure 3a,b). In adult blood, there were no differences in cytokine secretion between the nonstimulated control and the biofilms.

Secretion of IL-8 was significantly higher in cord as compared with adult blood when stimulating with both the PIA $(P<0.001)$ (Figure $3 a)$ and the non-PIA biofilm $(P<0.001)$ (Figure $3 b)$. A significantly higher IL-6 secretion was also observed in cord as compared with adult blood after stimulation with a PIA biofilm $(P=0.002)$, although in general the level of IL-6 secretion was low. No significant differences were found between PIA and non-PIA biofilms in the cord or adult blood.

\section{Anti-PIA and Anti-S. epidermidis IgG Titers in Nonstimulated Cord} Blood and Adult Blood

Significantly higher levels $(P=0.002)$ of anti-PIA IgG and anti-S. epidermidis $\operatorname{IgG}(P=0.001)$ were found in the adult as compared with the cord blood (Figure 4a,b).

\section{Complement Activation Induced by a PIA or a Non-PIA Biofilm in Cord Blood}

Both biofilms induced a significant activation of all complement factors as compared with the nonstimulated control (Figure 5). Activation of the $\mathrm{C} 1 \mathrm{rs} / \mathrm{C} 1$ inhibitor complex, which is specific for the classic pathway, indicates initial activation of the complement cascade through the classic pathway. There was also an activation of $\mathrm{C} 4 \mathrm{bc}$, which is common for both the classical and lectin pathways. The increase in $\mathrm{C} 3 \mathrm{bBbP}$ also indicates activation of the alternative pathway, probably through the alternative amplification loop.

The PIA biofilm (SE1457) induced a significantly higher activation of the $\mathrm{C} 1 \mathrm{rs} / \mathrm{C} 1$ inhibitor complexes $(P=0.008)$ and $\mathrm{C} 3 \mathrm{bc}(P=0.001)$ as compared with the non-PIA biofilm (M10) (Figure 5). The differences observed for the remaining activation product were all in the same direction (lower in the non-PIA biofilm) but did not reach statistical significance.

\section{DISCUSSION}

This is, to our knowledge, the first report on neonatal complement activation upon stimulation with SE biofilms. Previous studies in adults have suggested that the complement system is important in fighting biofilm-associated SE infections $(10,23)$. We found lower complement activation in the cord blood as compared with adult blood but a more powerful secretion of early proinflammatory cytokines. Biofilm-associated SE infections are commonly encountered in modern neonatal intensive care $(1,2)$. It is therefore important to understand the underlying pathogenic mechanisms in biofilm-associated SE infections in order to develop new treatment strategies. 


\section{Articles | Gransloet al.}
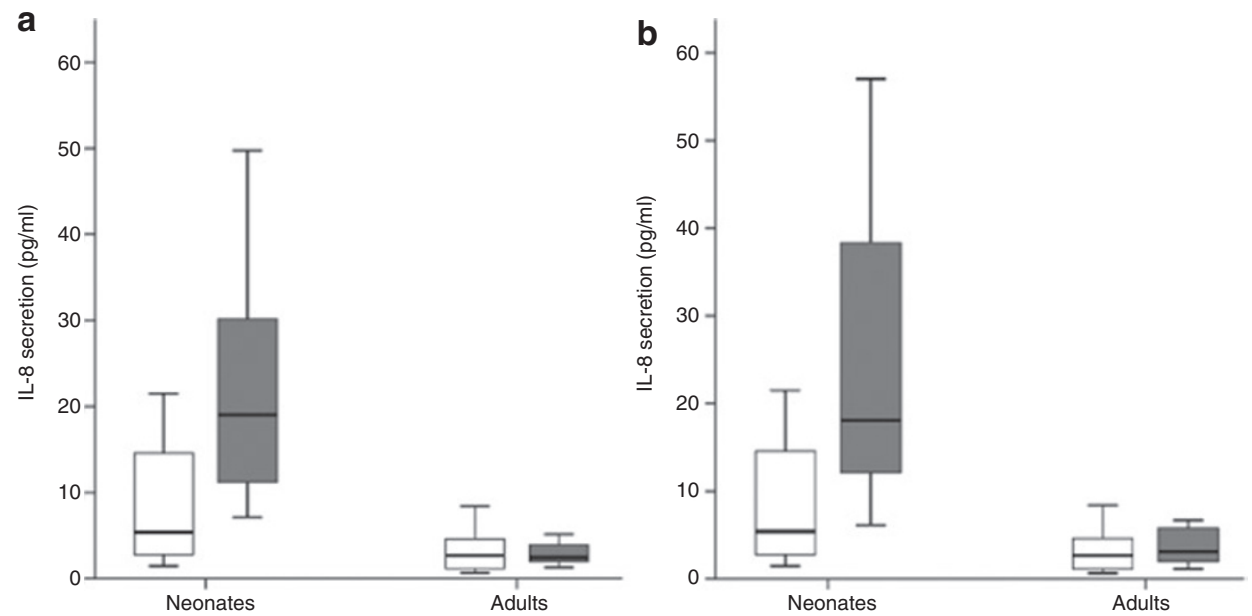

Figure 3. Comparison of the IL-8 secretion in umbilical cord and adult blood after incubation with (a) a PIA biofilm and (b) a non-PIA biofilm. The overall differences between neonate $(n=15)$ and adult $(n=6)$ secretion of IL-8 after incubation with (a) a PIA and (b) a non-PIA biofilm were statistically significant. Only the neonatal secretion was significantly higher than that of the nonstimulated control. White bars, nonstimulated sample; gray bars: (a) PIA-positive biofilm and (b) non-PIA biofilm. IL, interleukin; PIA, polysaccharide intercellular adhesin.

Both the PIA biofilm and the non-PIA biofilm induced higher complement activation in adult vs. cord blood. Our study indicates that the complement cascade was mainly activated through the classic pathway, although an increase in $\mathrm{C} 4 \mathrm{bc}$ may also reflect activation of the lectin pathway. The substantial activation in the alternative pathway probably reflects an activation of the alternative amplification loop $(24,25)$. In a previous study, we found the same pattern of complement activation in adults (23).

The classical pathway can be activated through different mechanisms: (i) direct binding of bacterial surface components to the first classic factor, C1q, (ii) binding through C-reactive protein, or (iii) opsonization by antibodies bound to antigens on the bacterial surface, such as PIA (26) or other antigens in the biofilm/on the bacterial surface. High PIA antibody titers have been reported both in patients with SE biofilm prosthesis infections and in healthy controls $(26,27)$. Due to the short incubation time in our experiments, any antibody-dependent complement activation relies on preexisting antibodies. IgG antibodies are actively transported across the placenta in the last trimester of the pregnancy. Yet total IgG level is markedly lower in neonates (cord blood) than in adults (28). Furthermore, differences in transport kinetics between the IgG subclasses may cause qualitative differences in titers of IgG subclasses (29,30), e.g., IgG1, IgG3, and IgG4 are fairly efficiently transported across the placenta, whereas transport of IgG2 is less efficient (30). It has previously been reported that preterm neonates have considerably lower titers of anticoagulase-negative staphylococci IgG antibodies and antibody-mediated opsonic activity against coagulase-negative staphylococci as compared with adults $(31,32)$. However, an association between level of anticoagulase-negative staphylococci antibodies and risk of neonatal coagulase-negative staphylococci sepsis has not been documented (32). PIA antibodies mainly belong to the IgG2 subclass $(26,33)$, which are modest complement activators. Our study showed that there are significantly lower antibody titers both toward the biofilm matrix (PIA) and toward the whole bacterium (S. epidermidis SE1457) in cord blood of term infants as compared with adult blood. Lower levels of antibodies, in addition to an inefficient IgG2 complement activation, might be one reason for lower classic complement activation in neonates vs. adults.

Both PIA and non-PIA biofilms were capable of activating the complement cascade in both neonates and adults. However, they induced significantly higher activation of the alternative amplification loop in adults as compared with neonates. One purpose of this alternative amplification loop is to increase the amount of activated complement factors in the final common pathway and thus increase the total activity of the complement system $(24,25)$. Neonates may have a deficient upregulation of the alternative pathway because of low levels of alternative pathway factors $(15,34)$. A lower activity of the alternative amplification loop may also explain why we observed lower levels of activation products of the final common pathway in cord blood. We have previously shown that PIA biofilm and purified PIA induced a strong complement activation (23). Similar findings were observed in the current study. The PIA biofilm induced markedly stronger complement activation than the non-PIA biofilm, both in adult and cord blood. Collectively, these data support our hypothesis that PIA is a complement activator $(10,23)$ in neonates as well.

We observed a higher secretion of both IL-6 and IL-8 in cord as compared with adult blood. Previous studies have indicated no difference in proinflammatory cytokine response between term neonates and adults after SE stimulation $(19,21)$. However, differences in the study design regarding incubation times and their use of planktonic SE instead of the biofilm used in our study may explain these differences. Tatad et al. showed that after stimulating monocytes for $18 \mathrm{~h}$ with SE, there was a significantly higher secretion of IL-6, IL-8, and IL-12 in adult blood as compared with blood from term neonates (35). It is possible that the adult cytokine secretion would reach the same level as that of neonates if longer incubation times had been used in our 
a

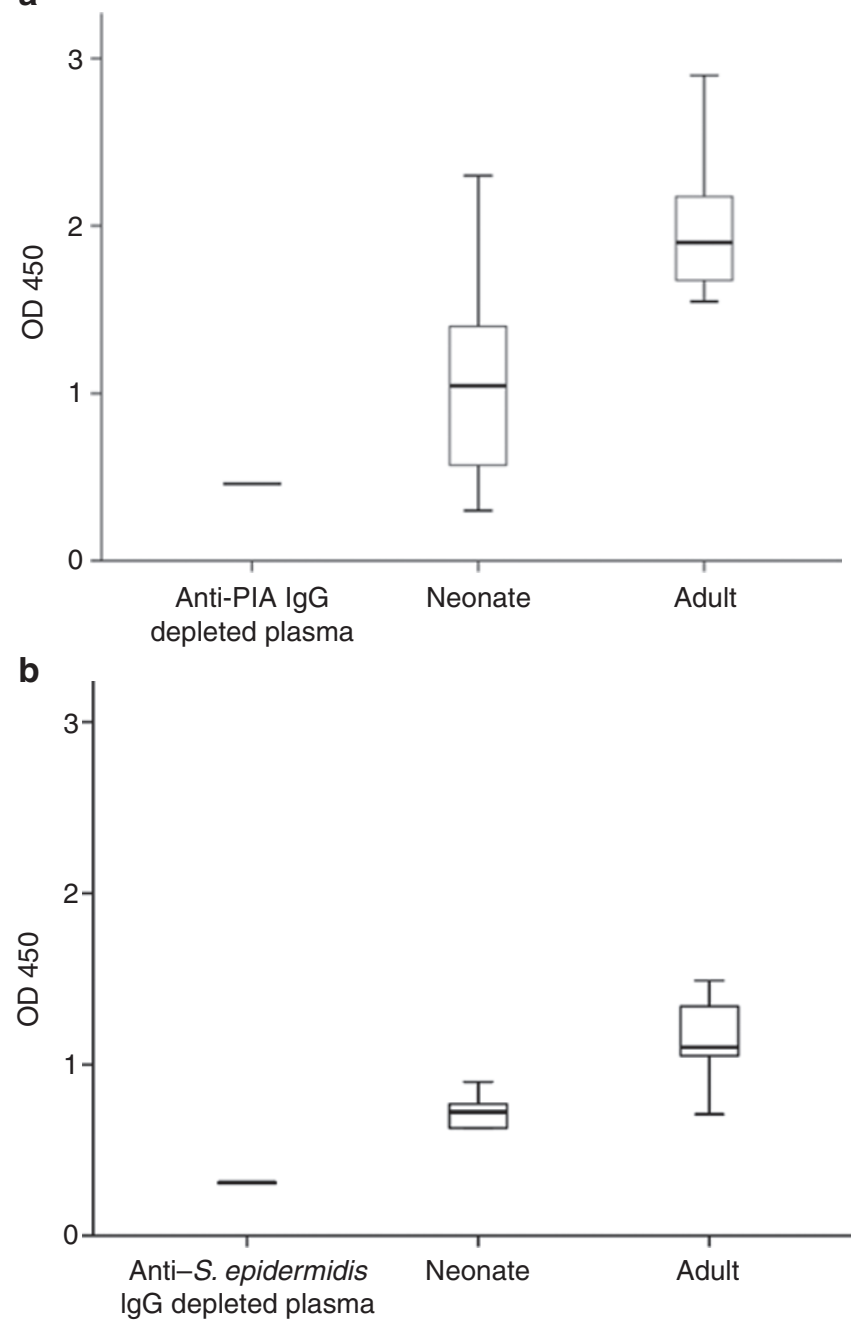

Figure 4. Comparison of (a) the anti-PIA IgG titers and (b) anti-S. epidermidis IgG titers (expressed as units of $\mathrm{OD}$ ) in the nonstimulated umbilical cord and adult blood. (a) Anti-PIA IgG and (b) anti-S. epidermidis IgG-depleted blood were used to set the cutoff for nonspecific lgG binding in the assay. The overall difference between neonates and adults was statistically significant for both anti-PIA IgG $(P=0.002)$ and anti-S. epidermidis IgG $(P=0.001)$. OD, optical density; PIA, polysaccharide intercellular adhesin.

study. Consistent with our findings, the complement response was lower and the cytokine response was more pronounced in a study in which meconium was incubated with neonatal vs. adult blood (22). This underscores the notion that the innate immune system in neonates is not immature in all branches of the inflammatory network, as also confirmed in this study.

The current study shows contrary results for the complement activation and cytokine response when comparing neonates and adults. In a previous study, we also found a "delicate balance" between the complement activation and cytokine secretion wherein high complement activation seemed to be accompanied by low cytokine secretion and vice versa (23). The interplay between complement activation and cytokine secretion is complex, with some cytokines being complement dependent whereas others are complement independent

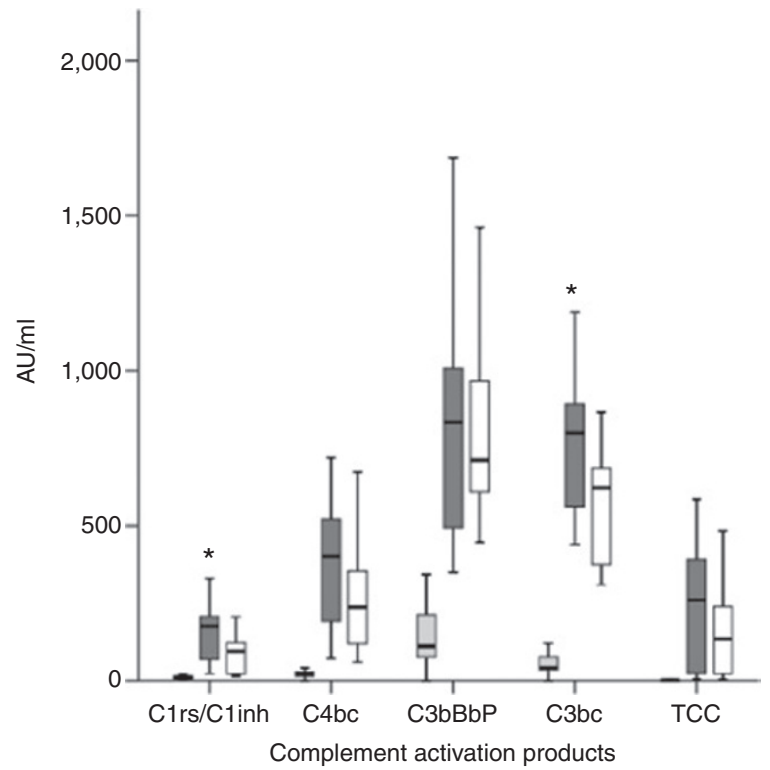

Figure 5. Comparison of the complement activation products in umbilical cord blood incubated with phosphate-buffered saline (nonstimulated), a PIA biofilm, and a non-PIA biofilm. The levels of the complement activation products $\mathrm{C} 1 \mathrm{rs} / \mathrm{C} 1$ inhibitor complex, C4bc, C3bBbP, C3bc, and terminal complement complex (TCC) were measured. Light gray bars, nonstimulated control; dark gray bars, PIA biofilm; white bars, non-PIA biofilm. *Statistically significant differences, $P<0.05$. PIA, polysaccharide intercellular adhesin.

$(36,37)$. Cytokines may, however, indirectly contribute to complement activation, e.g., by increasing the expression of anaphylatoxin receptors (38). High levels of C5a may also inhibit neutrophils (39) and regulate the transcription of cytokines $(40,41)$. The interplay between different parts of the innate immune response in neonates merits further investigation.

This study has limitations. First, the 30-min incubation time was a compromise to be able to analyze early activation of free circulating complement activation products (37). However, it may take hours before some of the proinflammatory cytokines are secreted in response to an infection. Thus, the current study describes only the initial cytokine response. Second, we included cord blood from term-born infants in this study. However, preterm infants represent the population "most at risk" for SE infections, and therefore future studies are needed to study this high-risk group. Third, we did not record the differential leukocyte counts for each experimental setup. We can therefore not totally rule out the possibility that quantitative differences in leukocyte counts may have influenced the inflammatory response. Fourth, the biofilm mass of the PIA biofilm was larger than that of the non-PIA biofilm (23). However, inflammatory response is not only positively correlated to biofilm mass. Indeed, a higher cytokine secretion and leukocyte activation was induced by a non-PIA biofilm (23). We therefore believe that our findings are not dependent on the biofilm mass but rather on differences in the activating potential of the components in the two biofilms. Finally, the ex vivo sepsis model enables us to study the host inflammatory response in whole blood, including detailed analysis 
of complement and cytokine secretion and their interaction. However, this model is not able to inform on the cooperation with other body tissue compartments, e.g., the endothelium surface. Thus, in vivo experiments are needed to include all components of the inflammatory system when assessing the pathogenic mechanisms of biofilm-associated SE infections.

\section{Conclusion}

We found a significantly lower complement response in neonates as compared with adults, when stimulating with both a PIA and a non-PIA SE biofilm. Our findings indicate a maturational deficiency of the neonatal complement system. This may decrease the ability of neonates to combat biofilm-associated SE infections. Further studies are needed to assess the role of the complement system in neonatal SE infections in order to develop potential new therapeutic strategies.

\section{METHODS}

\section{Bacterial Strains and Culture Conditions}

The PIA-producing S. epidermidis strain SE1457 and its isogenic mutant M10 were used. SE1457 is a clinical strain from an infected central venous catheter (41). M10 was created by insertion of Tn917 into the icaA gene of the ica-operon, obliterating PIA production $(41,42)$. We have previously shown that M10, when incubated in a glucoserich medium, produces a biofilm consisting of proteins and DNA (23). Biofilm formation in polyvinyl chloride (PVC) tubing (length $30 \mathrm{~cm}$, internal diameter $3 \mathrm{~mm}$, Mediplast, Malmø, Sweden) was performed as described previously (23). Briefly, overnight cultures in tryptic soy broth were diluted 1:100 in tryptic soy broth with $1 \%$ glucose and transferred to the PVC tubing segments. Each segment was closed end to end to form small loops. The loops were rotated slowly in an incubator at $37^{\circ} \mathrm{C}$ for $24 \mathrm{~h}$, cultures were emptied from the loops, and the loops were then carefully washed once with sterile phosphate-buffered saline. Production of biofilm in the loops was verified by staining the biofilm with crystal violet as previously described, showing that M10 produced a biofilm with a lower biofilm mass as compared with that of SE1457 (23). The loops with preformed biofilms were then stored at $-20^{\circ} \mathrm{C}$ until they were thawed immediately before the final experiments were carried out. A pilot study showed that the complement activation generated by "fresh" and "frozen-thawed" biofilm was identical; therefore, the complement-activating properties of the biofilm were not affected by freezing.

\section{Study Group}

Pregnant women were recruited from a study investigating maternal, placental, and fetal hemodynamics in low-risk pregnancies. A convenience sample of cord blood from 20 term-born neonates was initially included in this study. The umbilical cord was clamped immediately after birth, and blood was obtained from the umbilical cord. Five samples were later discarded due to signs of perinatal infection, small sample volume, or a delay of $>30$ min from delivery until the experiment was started. Thus, the final study group constituted cord blood from 15 infants ( 9 girls) with a median (range) birth weight of 3,678 $(2,898-4,360) \mathrm{g}$ and median (range) Apgar scores of $9(6-9)$ at $1 \mathrm{~min}$ and $10(7-10)$ at $5 \mathrm{~min}$ after birth. The median (range) umbilical cord $\mathrm{pH}$ and base excess were $7.27(7.14-7.40)$ and $-3(-7.2$ to 7.2$) \mathrm{mmol} / \mathrm{l}$, respectively. Venous blood samples from a total of six healthy adults (four women) were used as controls in this study.

\section{Ex Vivo Human Whole-Blood Model}

Cord blood and blood from adult controls were collected in sterile polypropylene tubes ( $5 \mathrm{ml}$ Nunc cryotubes; Nagle Nunc International, Naperville, IL) containing $50 \mu \mathrm{g} / \mathrm{ml}$ lepirudin (Refludan, CSL Behring, Marburg, Germany). Lepirudin, a recombinant hirudin analog with highly specific thrombin inhibitory activity, was used as an anticoagulant because it has no effect on complement activation (43). Induction of
Table 1. Complement activation products

\begin{tabular}{llc}
$\begin{array}{l}\text { Complement activation } \\
\text { products }\end{array}$ & Comment & Ref. \\
\hline $\begin{array}{l}\text { C1rs-C1 inhibitor } \\
\text { complex }\end{array}$ & Specific for the classic pathway & 45 \\
C4bc & $\begin{array}{l}\text { Common for the classic and lectin } \\
\text { pathways }\end{array}$ & 27 \\
C3bBbP & $\begin{array}{l}\text { Specific for the alternative pathway } \\
\text { C3bc }\end{array}$ & 37 \\
$\begin{array}{l}\text { Terminal complement } \\
\text { complex (TCC) }\end{array}$ & Cinal common pathway & 46 \\
\hline
\end{tabular}

inflammatory response in fresh whole blood was carried out as described earlier $(23,43)$, with the exception that only $900 \mu \mathrm{l}$ whole blood (cord blood and whole blood from adults) was used for each sample. Each experiment commenced within $30 \mathrm{~min}$ after delivery to avoid nonspecific activation of the innate immune system. All experiments were run in parallel with neonatal and adult blood using (i) sterile PVC loops (i.e., nonstimulated control), (ii) PVC loops with preformed SE1457 biofilm, and (iii) PVC loops with preformed SE1457-M10 biofilm. After the incubation period of $30 \mathrm{~min}$ at $37^{\circ} \mathrm{C}$, EDTA was added to a final concentration of $10 \mathrm{mmol} / \mathrm{l}$ to stop further activation. Plasma was then separated and stored at $-70^{\circ} \mathrm{C}$.

\section{Enzyme Immunoassays for Complement Activation Products}

Enzyme-linked immunosorbent assay (ELISA) was used to quantify central complement activation products (Table 1).

\section{Cytokine Multiplex Assay}

Multiplex assays for cytokines were used to study the secretion of four "early-response" proinflammatory cytokines (IL-6, IL-1 $\beta$, IL-8, and tumor necrosis factor- $\alpha$ ) in plasma. Analyses were performed by Bioplex cytokine assays (Bio-Rad, Hercules, CA) according to the manufacturer's instructions. The lower detection limits for the cytokines were $2.2 \mathrm{pg} / \mathrm{ml}$ for IL- $1 \beta, 1.8 \mathrm{pg} / \mathrm{ml}$ for IL-6, $1.4 \mathrm{pg} / \mathrm{ml}$ for IL-8, and 5.1 $\mathrm{pg} / \mathrm{ml}$ for tumor necrosis factor- $\alpha$.

\section{Purification of PIA}

PIA was prepared from a biofilm extract of a PIA-producing clinical strain, S. epidermidis CIP 109562, as previously described $(23,44)$.

\section{ELISA Determination of Anti-PIA IgG Titers in Nonstimulated Neonatal and Adult Blood}

The ELISA was performed as previously described (26). Briefly, Microlon 600 plates (Greiner-Bio, Frickenhausen, Germany) were coated with $100 \mu$ highly purified PIA at $1 \mu \mathrm{g} / \mathrm{ml}$ in $40 \mathrm{mmol} / \mathrm{l}$ sodium phosphate buffer, incubated overnight at room temperature, and then washed. The plates were blocked with $5 \%$ skim milk in Tris-buffered saline. Plasma was added at a concentration of 1:1,600 (diluted in Trisbuffered saline- $0.05 \%$ Tween 20 ) and incubated for $1 \mathrm{~h}$, washed with Tris-buffered saline- $0.05 \%$ tween 20 , and then incubated for $1 \mathrm{~h}$ with horseradish peroxidase-conjugated rabbit antihuman IgG (SigmaAldrich, St. Louis, MO), diluted 1:2,000 in Tris-buffered saline-0.05\% tween 20. Color was developed with $100 \mu \mathrm{l}$ of substrate solution (R\&D Systems, Minneapolis, MN) for $15 \mathrm{~min}$ in the dark, and then $50 \mu \mathrm{l}$ $\mathrm{H}_{2} \mathrm{SO}_{4}$ was added to stop the reaction. Optical density at $450 \mathrm{~nm}$ was measured. Background readings corresponding to the control wells incubated with blocking solution not containing sera were subtracted automatically. Anti-PIA IgG-depleted plasma was used as a cutoff marker for nonspecific IgG binding. Each experiment was conducted twice in triplicate. Antibody levels were expressed as units of optical density.

ELISA Determination of Anti-S. epidermidis IgG Titers in Nonstimulated Neonatal and Adult Blood

The ELISA was performed as previously described (32). Briefly, bacteria were killed by heat treatment $\left(56^{\circ} \mathrm{C}, 1 \mathrm{~h}\right)$ and washed with Hanks 
balanced salt solution (GIBCO Life Technologies, New York, NY). Polystyrene plates (Corning, New York, NY) were coated by adding 100 $\mu \mathrm{l}$ of bacterial suspension $\left(1 \times 10^{7}\right.$ colony-forming units $/ \mathrm{ml}$ in phosphate-buffered saline) and incubated at $37^{\circ} \mathrm{C}$ for $1 \mathrm{~h}$ and subsequently at $4^{\circ} \mathrm{C}$ overnight. Pilot studies showed that a plasma concentration of 1:3,200 is adequate. Antihuman IgG conjugate goat antihuman antibody (Promega, Madison, WI) diluted 1:2,500 in phosphate-buffered saline was used as secondary antibody. Optical density at $450 \mathrm{~nm}$ was measured. Background readings corresponding to the control wells incubated with blocking solution not containing sera were subtracted automatically. Anti-S. epidermidis IgG-depleted plasma was used as a cutoff marker for nonspecific IgG binding. Each experiment was conducted twice in triplicate. Antibody levels were expressed as units of optical density.

\section{Statistical Analyses}

The Mann-Whitney $U$ test was used to evaluate the differences in complement activation, cytokine secretion, and anti-PIA IgG and anti-S. epidermidis IgG titers between neonates and adults. The observed variations in adult complement activation and cytokine secretion were negligible when using blood from the same donor. To avoid problems with dependent observation among the adult controls, we used only the results from the first sample from each adult for the comparison with cord blood.

We performed parallel experiments with cord blood analyzing the responses upon exposure to the two biofilms. The immunological responses in each parallel experiment depend on individual properties of the blood, and we therefore used the nonparametric Wilcoxon's signed-ranks test to assess the level of significance. All tests were twotailed, and statistical significance was defined as $P<0.05$. All statistical analysis were performed using IBM-SPSS software, version 19.0 (IBM North America, New York, NY).

\section{Ethics}

The Regional Committee for Medical Research Ethics approved the collection of neonatal cord blood and blood from healthy adults for the immune response studies (REK number 2009/935-11). Informed written consent was obtained from the mothers of the neonates before delivery and from the adult control donors.

\section{ACKNOWLEDGMENTS}

We thank Grethe Bergseth and Judith Krey Ludviksen at the Research Laboratory at Nordland Hospital, Bodø, for excellent technical assistance; staff at the Department of Obstetrics \& Gynecology, University Hospital of Northern Norway, for help with recruitment and collection of samples; and the neonates, their parents, and the adult controls for their participation in the study.

\section{STATEMENT OF FINANCIAL SUPPORT}

This study was supported by grants from the Northern Norway Regional Health Authority.

\section{REFERENCES}

1. Stoll BJ, Hansen N, Fanaroff AA, et al. Late-onset sepsis in very low birth weight neonates: the experience of the NICHD Neonatal Research Network. Pediatrics 2002;110(2 Pt 1):285-91.

2. Isaacs D; Australasian Study Group For Neonatal Infections. A ten year, multicentre study of coagulase negative staphylococcal infections in Australasian neonatal units. Arch Dis Child Fetal Neonatal Ed 2003;88:F89-93.

3. Rupp ME, Fey PD, Heilmann C, Götz F. Characterization of the importance of Staphylococcus epidermidis autolysin and polysaccharide intercellular adhesin in the pathogenesis of intravascular catheter-associated infection in a rat model. J Infect Dis 2001;183:1038-42.

4. Rupp ME, Ulphani JS, Fey PD, Bartscht K, Mack D. Characterization of the importance of polysaccharide intercellular adhesin/hemagglutinin of Staphylococcus epidermidis in the pathogenesis of biomaterial-based infection in a mouse foreign body infection model. Infect Immun 1999;67:2627-32.

5. Stevens NT, Greene CM, O'Gara JP, Humphreys H. Biofilm characteristics of Staphylococcus epidermidis isolates associated with device-related meningitis. J Med Microbiol 2009;58(Pt 7):855-62.
6. Arciola CR, Gamberini S, Campoccia D, et al. A multiplex PCR method for the detection of all five individual genes of ica locus in Staphylococcus epidermidis. A survey on 400 clinical isolates from prosthesis-associated infections. J Biomed Mater Res A 2005;75:408-13.

7. Vuong C, Voyich JM, Fischer ER, et al. Polysaccharide intercellular adhesin (PIA) protects Staphylococcus epidermidis against major components of the human innate immune system. Cell Microbiol 2004;6:269-75.

8. Klingenberg C, Aarag E, Rønnestad A, et al. Coagulase-negative staphylococcal sepsis in neonates. Association between antibiotic resistance, biofilm formation and the host inflammatory response. Pediatr Infect Dis J 2005;24:817-22.

9. Cerca N, Jefferson KK, Oliveira R, Pier GB, Azeredo J. Comparative antibody-mediated phagocytosis of Staphylococcus epidermidis cells grown in a biofilm or in the planktonic state. Infect Immun 2006;74:4849-55.

10. Kristian SA, Birkenstock TA, Sauder U, Mack D, Götz F, Landmann R. Biofilm formation induces C3a release and protects Staphylococcus epidermidis from IgG and complement deposition and from neutrophil-dependent killing. J Infect Dis 2008;197:1028-35.

11. Clark LA, Easmon CS. Opsonic requirements of Staphylococcus epidermidis. J Med Microbiol 1986;22:1-7.

12. Levy O. Innate immunity of the newborn: basic mechanisms and clinical correlates. Nat Rev Immunol 2007;7:379-90.

13. Cheung GY, Otto M. Understanding the significance of Staphylococcus epidermidis bacteremia in babies and children. Curr Opin Infect Dis 2010;23:208-16

14. Wolach B, Dolfin T, Regev R, Gilboa S, Schlesinger M. The development of the complement system after 28 weeks' gestation. Acta Paediatr 1997;86:523-7.

15. Sonntag J, Brandenburg U, Polzehl D, et al. Complement system in healthy term newborns: reference values in umbilical cord blood. Pediatr Dev Pathol 1998;1:131-5.

16. Pillay V, Savage N, Laburn H. Circulating cytokine concentrations and cytokine production by monocytes from newborn babies and adults. Pflugers Arch 1994;428:197-201.

17. Strunk T, Temming P, Gembruch U, Reiss I, Bucsky P, Schultz C. Differential maturation of the innate immune response in human fetuses. Pediatr Res 2004;56:219-26.

18. Ng PC, Li K, Wong RP, et al. Proinflammatory and anti-inflammatory cytokine responses in preterm infants with systemic infections. Arch Dis Child Fetal Neonatal Ed 2003;88:F209-13.

19. Härtel C, Osthues I, Rupp J, et al. Characterisation of the host inflammatory response to Staphylococcus epidermidis in neonatal whole blood. Arch Dis Child Fetal Neonatal Ed 2008;93:F140-5.

20. Björkqvist M, Jurstrand M, Bodin L, Fredlund H, Schollin J. Defective neutrophil oxidative burst in preterm newborns on exposure to coagulasenegative staphylococci. Pediatr Res 2004;55:966-71.

21. Mohamed MA, Cunningham-Rundles S, Dean CR, Hammad TA, Nesin M. Levels of pro-inflammatory cytokines produced from cord blood invitro are pathogen dependent and increased in comparison to adult controls. Cytokine 2007;39:171-7.

22. Salvesen B, Fung M, Saugstad OD, Mollnes TE. Role of complement and CD14 in meconium-induced cytokine formation. Pediatrics 2008;121: e496-505.

23. Fredheim EG, Granslo HN, Flægstad T, et al. Staphylococcus epidermidis polysaccharide intercellular adhesin activates complement. FEMS Immunol Med Microbiol 2011;63:269-80.

24. Harboe M, Ulvund G, Vien L, Fung M, Mollnes TE. The quantitative role of alternative pathway amplification in classical pathway induced terminal complement activation. Clin Exp Immunol 2004;138:439-46.

25. Harboe M, Garred P, Karlstrøm E, Lindstad JK, Stahl GL, Mollnes TE. The down-stream effects of mannan-induced lectin complement pathway activation depend quantitatively on alternative pathway amplification. Mol Immunol 2009;47:373-80.

26. Sadovskaya I, Faure S, Watier D, et al. Potential use of poly-N-acetylbeta-(1,6)-glucosamine as an antigen for diagnosis of staphylococcal orthopedic-prosthesis-related infections. Clin Vaccine Immunol 2007;14: 1609-15. 
27. Wolbink GJ, Bollen J, Baars JW, et al. Application of a monoclonal antibody against a neoepitope on activated C4 in an ELISA for the quantification of complement activation via the classical pathway. J Immunol Methods 1993;163:67-76.

28. Ghoshal AK, Soldin SJ. Evaluation of the Dade Behring Dimension RxL: integrated chemistry system-pediatric reference ranges. Clin Chim Acta 2003;331:135-46.

29. Landor M. Maternal-fetal transfer of immunoglobulins. Ann Allergy Asthma Immunol 1995;74:279-83; quiz 284.

30. van den Berg JP, Westerbeek EA, van der Klis FR, Berbers GA, van Elburg RM. Transplacental transport of IgG antibodies to preterm infants: a review of the literature. Early Hum Dev 2011;87:67-72.

31. Fleer A, Gerards LJ, Aerts P, et al. Opsonic defense to Staphylococcus epidermidis in the premature neonate. J Infect Dis 1985;152:930-7.

32. Krediet TG, Beurskens FJ, van Dijk H, Gerards LJ, Fleer A. Antibody responses and opsonic activity in sera of preterm neonates with coagulasenegative staphylococcal septicemia and the effect of the administration of fresh frozen plasma. Pediatr Res 1998;43:645-51.

33. Kelly-Quintos C, Kropec A, Briggs S, Ordonez CL, Goldmann DA, Pier GB. The role of epitope specificity in the human opsonic antibody response to the staphylococcal surface polysaccharide poly $\mathrm{N}$-acetyl glucosamine. J Infect Dis 2005;192:2012-9.

34. Adamkin D, Stitzel A, Urmson J, Farnett ML, Post E, Spitzer R. Activity of the alternative pathway of complement in the newborn infant. J Pediatr 1978;93:604-8.

35. Tatad AM, Nesin M, Peoples J, et al. Cytokine expression in response to bacterial antigens in preterm and term infant cord blood monocytes. Neonatology 2008;94:8-15.

36. Brekke OL, Christiansen D, Fure H, et al. Combined inhibition of complement and CD14 abolish E. coli-induced cytokine-, chemokine- and growth factor-synthesis in human whole blood. Mol Immunol 2008;45:3804-13.

37. Mollnes TE, Brekke OL, Fung M, et al. Essential role of the C5a receptor in $E$ coli-induced oxidative burst and phagocytosis revealed by a novel lepirudin-based human whole blood model of inflammation. Blood 2002;100:1869-77.
38. Riedemann NC, Neff TA, Guo RF, et al. Protective effects of IL-6 blockade in sepsis are linked to reduced C5a receptor expression. J Immunol 2003;170:503-7.

39. Conway Morris A, Kefala K, Wilkinson TS, et al. C5a mediates peripheral blood neutrophil dysfunction in critically ill patients. Am J Respir Crit Care Med 2009;180:19-28.

40. Lappegård KT, Christiansen D, Pharo A, et al. Human genetic deficiencies reveal the roles of complement in the inflammatory network: lessons from nature. Proc Natl Acad Sci USA 2009;106:15861-6.

41. Riedemann NC, Guo RF, Hollmann TJ, et al. Regulatory role of C5a in LPS-induced IL-6 production by neutrophils during sepsis. FASEB J 2004;18:370-2.

42. Mack D, Nedelmann M, Krokotsch A, Schwarzkopf A, Heesemann J, Laufs R. Characterization of transposon mutants of biofilm-producing Staphylococcus epidermidis impaired in the accumulative phase of biofilm production: genetic identification of a hexosamine-containing polysaccharide intercellular adhesin. Infect Immun 1994;62: 3244-53.

43. Rupp ME, Ulphani JS, Fey PD, Mack D. Characterization of Staphylococcus epidermidis polysaccharide intercellular adhesin/hemagglutinin in the pathogenesis of intravascular catheter-associated infection in a rat model. Infect Immun 1999;67:2656-9.

44. Sadovskaya I, Chaignon P, Kogan G, Chokr A, Vinogradov E, Jabbouri S. Carbohydrate-containing components of biofilms produced in vitro by some staphylococcal strains related to orthopaedic prosthesis infections. FEMS Immunol Med Microbiol 2006;47:75-82.

45. Fure H, Nielsen EW, Hack CE, Mollnes TE. A neoepitope-based enzyme immunoassay for quantification of C1-inhibitor in complex with C1r and C1s. Scand J Immunol 1997;46:553-7.

46. Mollnes TE. Analysis of in vivo complement activation. In: Herzenberg LA, Weir DM, eds. Weir's Handbook of Experiental Immunology. vol. 78. Boston, MA: Blackwell Science, 1997.

47. Mollnes TE, Lea T, Harboe M. Detection and quantification of the terminal C5b-9 complex of human complement by a sensitive enzyme-linked immunosorbent assay. Scand J Immunol 1984;20:157-66. 